\title{
Konsilien von Professoren aus Neapel und Padua gegen die Sehstörungen von G. V. Pinelli*
}

Von Luigi Belloni

Der «Fondo Pinelli» der Mailänder Ambrosiana-Bibliothek ${ }^{1}$ ist den Wissenschafts- und Medizinhistorikern seit geraumer Zeit als eine ausgiebige Quelle interessanter unveröffentlichter Schriften wohlbekannt.

Gian Vincenzo Pinelli (1535-1601) wurde in Neapel geboren und stammte aus einer Familie genuesischer Herkunft. 1558 siedelte er nach Padua über, wo er bis zu seinem Tode blieb. Reich an Familiengütern, wurde es einem typischen Gelehrten des Cinquecento wie Pinelli ermöglicht, seine vielseitige Wißbegier und seine enzyklopädischen Interessen völlig zu befriedigen. In seinem gastfreundlichen Hause in Padua verkehrten viele der bedeutendsten Gelehrten seiner Zeit.

Seine Bibliothek - die nach Pinellis Tode an die Ambrosiana überging ist reich an Miszellen, worin er nicht nur seinen eigenen Briefwechsel, sondern auch die von Freunden und Bekannten geschriebenen oder erhaltenen Briefe, Abhandlungen, Dissertationen usw. im Original oder in der Abschrift sammelte, die irgendwie seinen unaufhaltsamen Wissensdurst löschen konnten.

Ich hatte schon Gelegenheit, aus dem «Fondo Pinelli» zu schöpfen ${ }^{2,3,4}$ : und bei einem solchen Anlaß ${ }^{5}$ - bei dem ich mich in Wirklichkeit um einen ganz anderen Gegenstand bemühte - konnte ich ein Konsilium gegen Pinellis Sehstörungen ausfindig machen. Ich habe dieses Konsilium mit zwei weiteren desselben «Fondo Pinelli» in Verbindung gebracht, die ebenfalls von Sehstörungen handeln. Obgleich hier der Name des Patienten fehlt, fühle ich mich dazu berechtigt, diese Störungen mit denen Pinellis zu identifizieren.

Als erstes bringe ich das Konsilium von Iulius Iasolinus (1537-1622), das außer dem Datum (Neapel, den 1.Februar 1581) auch den Namen des Patienten, für den es geschrieben war, aufweist. Paul Buchner (1886-1978) ${ }^{6}$ widmete Iasolino, Professor der Anatomie und Chirurgie an der Universität von Neapel und Verfasser eines grundlegenden Werkes über die Thermen der

* Beitrag zur Festschrift für Professor Heinrich Buess, Basel.

Gesnerus 1/2 (1981) 


\title{
Insel Ischia ${ }^{7}$, eine vorzügliche Ergobiographie. Mit Ausnahme der Unter- schrift ist das Konsilium nicht eigenhändig von Iasolino:
}

\author{
ms. Ambros. $S 80$ sup., c. 76
}

Quantum ex his quae scripta et relata sunt, coniicere potui, casus Illustris ac Praeclarissimi D. Ioannis Vincentii Pinelli, sunt capitis imbecillitas, et diminutio et quasi ablatio visus: namque scribitur quod pusilli quidam culices, ac caliginosa quaedam oculis saepe illi obversari videntur: auctoque malo, augescunt iam dicta symptomata. Adeo denique affectio increvit, ut aegrotanti absque crebra intermissione neque legere, neque scribere fas sit. Quartus agitur fere mensis cum haec affectio laborantem excruciat. Verum hae suffusionis notae sunt, inchoantis tamen mali, ut Aetio ${ }^{8}$ aliisque doctis Medicis visum fuit. Liquet etiam et ex his, quod cum divulsa, nec inter se coniuncta, crassa corpora fuerint, quae iuxta pupillam consistunt, fluitentque in tenui humore, tanquam culices quidam foris obversari videntur, iis qui sic se habent. Ab ore ventriculi malum procedere haud puto. Scribitur enim tribus quatuorve, ut diximus, mensibus, perpetuo symptomata apparere, et quod singulis diebus huiuscemodi apparitio perseveravit, nullo interveniente die, qui omni querela caruerit: haec assiduitas suffusionem portendit.

At quoniam morbus hic, non in totum est factus, sed adhuc fit: vel si non fit, periculum est ne adhuc crescat, ideo praeservativo etiam genere utendum, imo ab eo incipiendum: frustra enim materiam illam quae morbum peperit amovemus, si alia loco eius semper praesto sit. Cavendum igitur ne materia adsit, quae ad locum possit defluere: congruo igitur victu procurandum, ut neque de integro generetur: vel, siqua, genita est, vel generabitur, vacuanda, et alio distrahenda est. Et quia ex imbecillitate cerebri congregatur, roborandum cerebrum, ne eam congreget: roborandi et oculi ne recipiant. Erunt quatuor in genere intentiones praeservativae: ratio victus, vacuatio, diversio, membrorum roboratio. Curativa unica: vacuatio. Quibus vero praesidiis haec molienda, morosus fuerim, ne dicam temerarius, si praesentes medicos doctrina et experientia percelebres docere audeam: illudque in me merito dicetur, Noctuas Athenas.

Tantummodo igitur aliqua signabo naturalia praesidia, quae in nostris tantum regionibus reperiuntur, talibus affectionibus sacrata. Primatum autem obtinet fumus aquae sulphurariae in Puteolis: visum acuit, rheuma, frigusque capitis tollit, ipsumque roborat. Praeterea aquam bullae sudatoriae et Puteolanum aerem mirificam ad hos pellendos affectus vim habere quotidie experimur. In Aenaria Insula ${ }^{9}$ est balneum Dimidiae viae, et Plagae Romanae, atque aliud a nobis nuper repertum iuxta Gurgitelli aquas, oculare dictum. His praesidiis aegrotantes plures iisdem affectionibus, et saevioribus, pristinae sanitati restituimus: absque alia medica ope. Et quia morbus magnum minatur malum: statim ideo illi naturalibus, expertisque praesidiis, quibus fidere oportet, occurrendum erit. Commendarem igitur summopere Aegrotantis accessum ad praecitata loca: verno tamen tempore, quod instat ac fere prope est. Verum si accessum aegrotus recusabit: nullo pacto cauterium vitabit, et praesertim in brachio, si primo a partibus magis distantibus incipiendum videbitur.

Curativam intentionem aliter non prosequor, sed ipsam, atque etiam quaecumque 
superius scripsimus, iudicio et arbitrio Praestantissimorum praesentium Medicorum relinquimus: quibus me plurimum opto esse commendatum.

Neapoli Kalendis februarij Salutis Dn: Anno M.D.lxxxj.

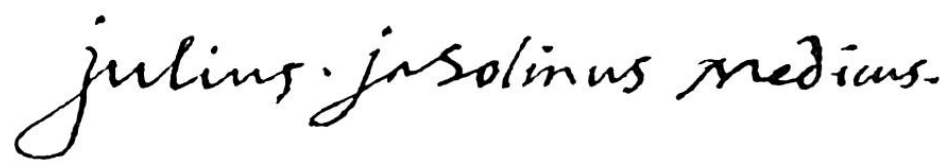

Etwa ein Drittel des Konsiliums von Iasolinus ist den Thermalkuren nicht nur der Insel Ischia, sondern auch von Pozzuoli gewidmet. Letztere werden im Konsilium des anderen neapolitanischen Professors empfohlen, d.h. in dem von Giovanni Bernardino Longo (gest. 1599) ${ }^{10}$, das «eigenhändig geschrieben» («scritto di sua mano») ${ }^{11}$ und demzufolge sehr schwer zu lesen ist, obschon unter neapolitanischen Einflüssen die italienische Sprache anstelle des Lateins angewandt ist. Immerhin wurde mir die Lektüre durch eine zeitgenössische, deutliche, wenngleich nicht immer zuverlässige Transkription erleichtert. Wenngleich der transkribierte Text damals schon an verschiedenen Stellen durch eine andere Hand korrigiert worden ist, sind dennoch einige deutlich lesbare Wörter zurückgeblieben, deren Bedeutung unklar ist:

\section{ms. Ambros. $S 80$ sup., cc. $77-78$ und c. 136}

Lassando di ricercare le cause, quale sono note appresso a tutti Medici, dico che essendo questo Signore nel capitulo detto de Immaginibus che appareno avante agli ochi, quale dependino da humori, o existenti tra le tunice del ochio o da vapori elevati dalle parte inferiori, quali sono causati per la debilità del stomaco, et poi il calor del fecato come agente fa la evaporatione, et lo cerebro debile recepe: et per lo continuo studio, la potentia visiva, per vider sempre cose contrarie bianche et negre, se debilita. Et si detto humore o vapore se interponesse nello forame del uvea ditto pupilla, se causerìa la suffusione ditta vulgarmente cataratta, dalla quale deve ditto Signore evitarsi quanto sia possibile. Adonque questo simptoma, che è diminuta visione, o depravata, o simili, segue il suo morbo, quale è la debilità, o intemperie del cerebro: et per questo, poi che ogne piccola actione lesa nelli occhi è de importanza, per esser senso più nobile et allo comodo della vita necessario, il mio parere è che subito se rimedia, avante che venga la suffusione: et prima, perchè non se può purgar nè sanar la parte, si primo non se sana il tutto, se deve purgar il corpo (pretermissa ogni evacuazione de sangue) con preparare le materie con lo rodomele, con lo syrupo de infusione de rose con acqua de betto, finochie di eufragia, et poi purgar il corpo con le pillole auree, lucis ${ }^{12}$, agarico trochiscato, sinequibus ${ }^{13}$ arabice secondo parerà al presente Medico: di là a doi hore con darli tre onze del syruppo delle nove infusione di rose rosse ditto solutivo, poi bisognierà venire subito alla fontanella, quale la farìa al sinistro 
braccio o destro: ma al sinistro per esser meno exercitato: non farìa nè lazo nè fontanella nella coppa ${ }^{14}$, perché penserìa debilitarsi il cerebro; ma nel braccio havriamo la diversione senza debilitare il cerebro, per non esser cossì da vicino.

E perchè bisogna non solo evacuare et divertire, ma confortare la parte imbecilla, quale non agrega iterum detti humori e li condensi nella parte affetta, sono de parer, che il più saluberrimo remedio porrìa partirse da questo loco ubi diu permansit secundo Hippocrate et venire alla zolfatara et aere Puteolani, perchè conforterebbe tanto la testa, il stomaco, consumaria li grossi humori, vivificarrebbe la vista e potentia visiva et l'organo, che io ne spererìa salute, sì per ragione, sì per continua experienza, quale ogne dì se vide: et farìa de più il sudatorio de Tritoli ${ }^{15}$, quale è de mirabile effetto a questo caso, il bagno del aqua de finochi. Lasso li dui rimedij, quali scrive la Scola de Medici, poichè questo Signore sta in parte dove non mancano nè eccellenti Medici nè rimedij.

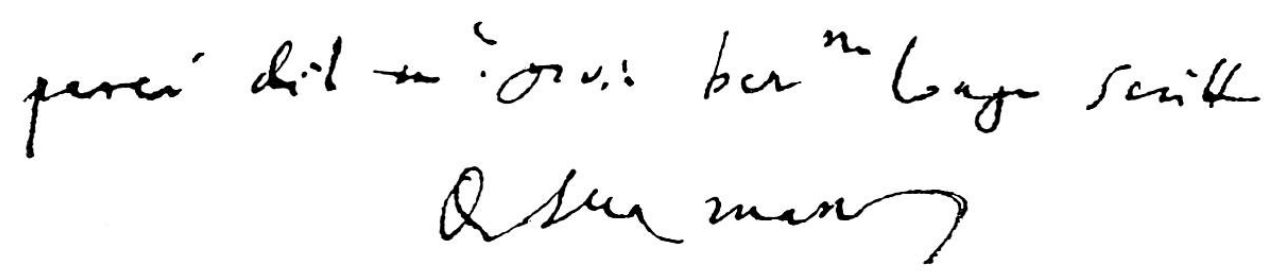

Am längsten ist das kollegiale Konsilium von drei Professoren der Universität Padua: Hieronymus Capivaccius (gest. 1589), Hieronymus Mercurialis $(1530-1606)^{16}$ und Hieronymus Fabricius ab Aquapendente (ca.15331619) ${ }^{17}$. Mit Ausnahme der Unterschriften der drei Verfasser ist das Konsilium von einem Amanuensis mit einigen (nicht korrigierten) Fehlern geschrieben $^{18}$ :

ms. Ambros. D 332 inf., cc.59-61

Vitium quo valde turbatur vir gravissimus et illustris, nihil aliud est quam visio tum imminuta, tum depravata: imminuta quidem, quoniam ne octo quidem versus constanter et commode aut legere aut scribere potest; depravata vero, cum imagines quamplurimae et diversorum generum continuo ante oculos obversari videantur: vi quorum malorum perterritus, nihil aliud studet, quam ut si fieri potest ab imminenti cecitate praeservetur. Id quod ut ex arte fieri possit, horum malorum causae sunt nobis perquirendae, quae non in facultatem visoriam, non in medium sunt referendae, neque in instrumenta ${ }^{19}$ : quare ex necessitate sunt referendae in ea quae sunt extra oculi constitutionem, nempe in spiritus visorios et materiam excrementitiam. Etenim cum ad exactam et perfectam visionem celebrandam praeter alia desideretur spiritus visorius lucidus, splendidus, purus, et qualis aether fulgidus nec non copiosissimus, ut sui multitudine quae longissime absunt conspiciantur, neque visio tam cito veluti defatigetur, et sui splendore perfecte aerem 
depurando obiecta discernantur: mirum non est si ob paucitatem spirituum atque eorundem impuritatem, visio in legendo quam citissime veluti defatigetur, et obiecta veluti caligine quadam obducta intueatur. Quod vero materia quaedam melancholica a praedominio tamen pituitosa, in humorem albugineum collapsa, faciat ut spectra rerum ob oculos volitantium conspiciantur, ex hoc facile concitari potest: nam cum aer et humores a spiritu visorio fuerint collustrati et cognationem ac familiaritatem quandam inter se contraxerint, quidquid in his fuerit coloratum, cum haec omni colore careant, sive illud sit in humoribus ipsis, sive in aere, speciem producit, quae in aere et in medio suscepta conspicitur: Cum itaque in aere appareant floci, aranearum telae et id genus aliae imagines, neque tamen re vera extra oculum existant; nunc est ut dicamus illarum imaginum corpora in oculo residere, praesertim cum perpetuo appareant et maxime cum aer ab utroque lumine, tum interno, tum externo, fuerit apprime collustratus ac depuratus. Diximus autem materiam hanc maiori ex parte pituitosam esse, quamvis subnigra conspiciatur, quoniam ad inficiendum colorem album exigua portio nigri satis esse videtur: neque putamus materiam hanc in oculo generari, quoniam si tanta esset partium oculi intemperies, ut haec materia generaretur, proculdubio suffusio eam esset subsecuta: consentaneum est itaque ut a capite in hanc partem collabatur, quae cum ab initio fuerit vaporosa, nunc concreta humorosa est iudicanda, non augetur tamen, quoniam etsi vapores ei deferantur, vi tamen ipsorum spirituum et caloris innati facile dissipantur: suscipitur autem ob ipsius oculi imbecillitatem, tum nativam ob malam conformationem, tum adscititiam propter insignem alterius oculi noxam ${ }^{20}$, nec non propter vigilias, labores et eximium doctrinae studium et singulare. Aggregatur autem eiusmodi materia in capite: et quia in eo generatur ob intemperiem illius humidiorem et in activis qualitatibus frigidiorem potius quam calidiorem; et quia vi caliditatis iecinoris a partibus infernis sursum in caput feruntur vapores, tum pituitosi, tum melancholici, ut mirum non sit si spiritus quoque visorii aliqua ex parte impuri reddantur, neque ita copiosi generentur quemadmodum ad perfectam et absolutam visionem desiderari videntur.

Quare ut praestantissimus vir ab imminenti periculo praeservetur, danda erit opera, ut, universo corpore purgato, caput quam diligentissime mundum reddatur, moderate calefiat, et maiori studio exiccetur, ut iecur refrigeretur, ita tamen vero ventriculus nullam laesionem patiatur; et materia in oculo existens discutiatur ac penitus dissipetur, et pro viribus studendum erit, ut oculus ipse ita corroboretur, ut vapores non ita facile suscipiat, et si susceperit, illius robore discutiantur ac dissipentur. Verum quia vix fieri poterit ut caput adeo confirmetur, quin continuo nova materia generetur, et infernae partes ita corrigantur quin ab his nonnihil sursum feratur, ob id tutius esse arbitramur, si omni studio, cura et diligentia procuremus, ut materia quae in capite generatur in proximam aliquam partem derivetur, et quae sursum fertur, deorsum revellatur, ut tandem caput ratione auxiliorum genere confirmatum et robustius redditum alimentum melius concoquat et propterea excrementa in minori copia generentur, et quae generantur, eoque feruntur aliunde vi expultricis per loca idonea et a natura comparata excernantur, ut inde spiritus et copiosiores et puriores generentur.

Quod quidem negotium quamvis arduum ac difficile videatur, nihilominus ea est viri praestantissimi prudentia, ea est illius autem constantia, ut non sit dubitandum, quin omnia ex voto succedant, etsi materia in oculo existens omnino extirpari ac penitus dissipari non poterit, illud saltem praestabimus, ac Dei beneficio re vera efficiemus, ut ab 
imminenti periculo praeservetur, et in dies melius et exactius videat, neque ita facile ex longa lectione patiatur.

Hoc autem ut consequamur auxiliorum materiae sunt proponendae, quarum cum aliae ad fontem chirurgicum, aliae ad pharmaceuticum, aliae ad diaeteticum pertineant: primo loco de materiis, seu instrumentis chirurgicis sermonem habebimus, ex quibus cum sola derivatoria et revulsoria hic locum habeant, haec tamen nobis erunt proponenda. Quare quod ad revulsoria spectat, frictiones opportunae iudicantur, quae ita administrari debent, ut prius fiant in cruribus, deinde in coxis, et sic paulatim ascendendo usque ad scapulas et brachia: quae tempore matutino administrari debent.

Quo vero ad derivatoria pertinet, cum melius, promptius et citius fiat derivatio per loca proximiora, quam per remotiora, ob id laudamus, imo nullum remedium praestantius esse putamus, quam si fiat setaceum in occipitio cum primum corpus fuerit expurgatum, quod quidem setaceum, cum caput fuerit aliquantulum confirmatum et ab imminenti periculo tuti erimus, tunc per cauterium in brachio excitatum submoveri poterit.

Verum cum antequam propositae materiae administrentur, corpus sit prius purgandum: ob id praemissa alvi lenitate vel cum cassia, vel ellectuario lenitivo, vel syrupo et melle rosato solutivo, vel manna, praeparabitur materia vel cum brodiis alteratis cum cicorea, betonica et modico feniculi, vel ipsis aliquid syrupi de cicorea atque de betonica commisceri poterit vel aliquid mellis rosati simplicis: post haec purgabitur corpus vel cum diluto agarici epithemate et nonnihil rhabarbari, cui addatur aliquid mellis rosati solutivi et manna vel syrupus rosarum solutivus, vel si pillulis magis gaudebit, maxime convenient pillulae cochiae, vel de tribus, vel aliae id genus; et si unica purga satis esse non videbitur, repetatur.

Corpore purgato, ad absumendas materias excrementitias non solum in toto, verum etiam in parte existentes, nihil utilius fore putamus quam decoctum salsae vel chinae, si cum carne et modico ligni et ocularibus praeparetur, ita tamen correctum et alteratum, ut iecur non incalescat.

Interim caput erit purgandum non masticatoriis, quoniam dentes facile patiuntur, non errhinis seu sternutatoriis, ne ex capitis concussione materia maiori cum impetu ad oculos praecipitetur; quare nares tamen inungantur oleo amigdalarum dulcium una cum butirro in decocto malvae optime loto, vel cum his quae vim habent liquandi materias absque mordicatione: quod si pro masticatoriis utamur gargarismate aliquo vel eclegmate ${ }^{21}$ non erit fortasse $\mathbf{p} . . .2^{22}$

Ut vero caput exiccetur et moderate calefiat non improbamus lotiones, quibus uti consuevit, si tamen parentur cum lixivio alterato cum his quae vim habent exiccandi et mediocriter calefaciendi, veluti est betonica, agaricus, folia salicis, radices filicis maris, scabiosa, et id genus alia quorum sylva est magna.

Ut vero oculus roboretur cum moderata abstersione, non erit ab re si administretur aqua panis cum feniculi seminibus et pulvere euphragiae parati: vel oculo cum vino aromatico albo roborato, si sieph album sine opio administretur; quod si sola roboratione contenti erimus, solo vini lavacro uti debemus. De sero lactis caprini, hac peracta medicatione, sermonem habebimus.

Fontes, aquas et vaporaria quae proponuntur a viris Excellentissimis ${ }^{23}$, nec probamus, nec improbamus, cum sola experientia nitantur.

His omnibus diligens victus ratio erit iniungenda, qua in re cum nullus a viro prudentis- 
simo committatur error, propterea non est ut in hac praescribenda multum temporis sumamus: illud tamen non videtur silentio praetermittendum plurimum prodesse posse vinum aliquod medicatum: et quoniam hoc tempore non ita commode parari potest, propterea opportunum erit si alteretur aqua cum praedictis ocularibus, et cum ea vinum diluatur.

Atque in hac tota medicatione suademus ut quantum fieri potest ab assidua lectione caveat et ab omni oculorum defatigatione.

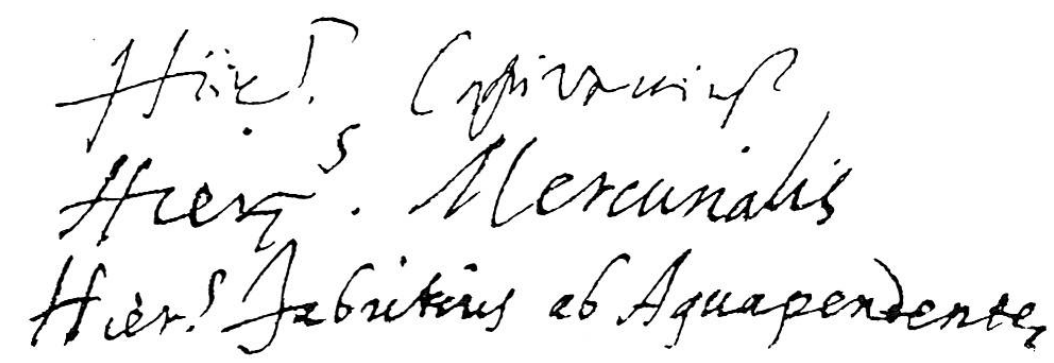

Manchmal, aber nicht immer, wurde die Schreibweise und die Anwendung der großen Buchstaben und der Absätze modernisiert; auch einige Verschreibungen (lapsus calami) wurden verbessert. Dies zum leichteren Verständnis des Lesers und um so mehr, da die veröffentlichten lateinischen Texte nicht eigenhändig geschrieben sind.

\section{Anmerkungen}

1 Adolfo Rivolta, Catalogo dei Codici Pinelliani dell'Ambrosiana, Mailand 1933.

${ }^{2}$ Luigi Belloni, Di una non avvenuta chiamata di Gaspare Tagliacozzi allo Studio di Padova (1594) e di un consulto epistolare tra G. Mercuriali, G. Tagliacozzi e G. Fabrici d'Acquapendente sovra un caso di fistola retto-genitale. In: Rivista di Storia delle Scienze mediche e naturali 43 (1952) 345-351.

${ }^{3}$ Luigi Belloni, Die deutsche Aussprache in einer kurzen Abhandlung von Conrad Hofmann an Hieronymus Fabrici ab Aquapendente. In: Sudhoffs Archiv für Geschichte der Medizin und der Naturwissenschaften 37 (1953) 201-209 (Diepgen-Festschrift).

${ }^{4}$ Luigi Belloni, Streitfragen zwischen Bartolomeo Eustachi und Gerolamo Mercuriali auf dem Gebiete der medizinischen Philologie. In: Gesnerus 33 (1976) 188-208.

${ }^{5}$ Belloni (zit. Anmerkung 3), Anmerkung 4, S. 203 f.

${ }^{6}$ Paolo Buchner, Giulio Iasolino, Medico calabrese del Cinquecento che dette nuova vita ai bagni dell'isola d'Ischia, Mailand 1958.

${ }^{7}$ Giulio Iasolino, De rimedi naturali che sono nell'isola di Pithecusa; hoggi detta Ischia ... Nelli quali si dimostrano molti rimedi naturali, dal detto Autore nuovamente ritrovati, oltre quelli che lasciarono scritti gli Antichi ..., Neapel 1588.

${ }^{8}$ Lib. VII, cap. 51 («De suffusione»). 
9 Über die einzelnen hier erwähnten Thermen der Insel Ischia verweise ich auf das in Anmerkung 7 angegebene Buch von Iasolino.

${ }^{10}$ Bartolomeo Chioccarelli, De illustribus scriptoribus qui in civitate et regno Neapolis ab orbe condito ad annum usque MDCXXXXVI. floruerunt, Bd.1, Neapel 1780, S. 320.

Camillo Minieri Riccio, Memorie storiche degli scrittori nati nel regno di Napoli, Neapel 1844, S. 182 .

Giovanni Bernardino Tafuri, Istoria degli scrittori nati nel Regno di Napoli, Neapel 1744-1770, 3/2, S. 495.

Nicola Toppi, Biblioteca Napoletana, et apparato de gli huomini illustri in lettere di Napoli, $e$ del Regno, Neapel 1678, S. 142 und 345.

11 "parer' del $\mathrm{m}^{\mathrm{o}}$ gio: ber ${ }^{\text {no }}$ longo scritto/di sua mano» (am Ende des Konsiliums photographisch reproduziert).

12 Pilulae lucis Mesuae.

${ }^{13}$ Pilulae sine quibus esse nolo Nicolai Salernitani.

${ }^{14}$ Genick.

15 Über das Balneum Trituli und das gleichnamige Sudatorium, heute «Stufe di Nerone» genannt, vgl. Petrus de Ebulo, Nomina et virtutes balneorum seu de balneis Puteolorum et Baiarum ... Introduzione di Angela Daneu Lattanzi, Rom 1962, S.91.

${ }^{16}$ Vgl. auch meine in Anmerkung 2 und 4 erwähnten Artikel.

17 Vgl. auch meine in Anmerkung 2 und 3 erwähnten Artikel.

${ }^{18}$ Eine lücken- und oft fehlerhafte italienische Übersetzung wurde von Aldo Bottero, Un consulto oculistico del Cinquecento, in Castalia 4 (1948) 189-192, veröffentlicht.

${ }^{19}$ Es folgen zwei offensichtlich bedeutungslose Wörter von je drei Buchstaben (per que).

${ }^{20}$ Nach Rivolta (zit. in Anmerkung 1), S.XXIX, «war - infolge eines Defektes am rechten Auge, das während eines kindlichen Streites durch seinen Bruder verletzt worden war Pinellis Sehkraft bedeutend geschwächt».

${ }^{21}$ Eclegma, d.h. eclectos, linctus, loch.

${ }^{22}$ Unleserliches Wort mit Ausnahme des Anfangsbuchstabens.

${ }^{23}$ Wie etwa Iasolino und Longo in den zwei vorausgegangenen Konsilien.

\section{Summary}

Gian Vincenzo Pinelli (1535-1601) was born in Naples and lived as a scholar at Padua. His library and much letters in manuscript, which he left behind, form the "Fondo Pinelli" in the Ambrosiana at Milan. Pinelli was afflicted with troubles of his eyesight. Here are communicated three consulting letters of medical professors about eatment of his eye-disease: one by Giulio Iasolino in Naples, 1581, one by Giovanni Bernardino Longo, also in Naples, and the third by three professors of Padua: Capivaccio, Mercuriali, and Fabrici d'Acquapendente.

Prof. Dr. Luigi Belloni

Istituto di Storia della Medicina

Università degli Studi

Via Festa del Perdono, 7

I-20122 Milano (Italien) 